\title{
Ultrastructural Development of Nostoc muscorum A
}

\author{
By ROCHELLE GINSBURG AND N. LAZAROFF \\ Department of Biological Sciences, State University of New York, \\ Binghamton, New York I390 I, U.S.A.
}

(Received 19 June 1972; revised 20 September 1972)

\begin{abstract}
SUMMARY
The ultrastructural development of Nostoc muscorum a was studied in preparations grown in darkness and sampled during succeeding intervals in the light. The major effect on ultrastructure during light-induced development is a re-organization of the thylakoid lamellae. The lamellar pattern in dark-grown aseriate organisms differs from that displayed by those grown in the light. Transfer of dark-grown algae to light results in breakdown of the original lamellar complement and formation of parietal whorls of lamellae. Within the dark-grown aseriate packets, some of the component cells which are indistinguishable in light microscopy can be identified ultrastructurally as immature heterocysts. Maturation of the heterocysts occurs after transfer to the light.
\end{abstract}

\section{INTRODUCTION}

The development of the filamentous blue-green alga, Nostoc muscorum A, is characterized by a cyclic sequence of morphological changes. A technique for the synchronization and subsequent controlled development of this organism has been described by Lazaroff \& Vishniac (196I). This approach has been used for investigating the ultrastructure of $N$. muscorum A undergoing morphological differentiation.

Several studies have indicated that the ultrastructure of blue-green algae may be strongly affected by conditions of illumination. Allen (1968) found the abundance of lamellae in Anacystis nidulans to be inversely proportional to light intensity. Findley, Walne \& Holton (I970) noted marked differences in lamellar structures of Chlorogloea fritschii cultured at various light intensities. Peat \& Whitton (1967) observed that vesiculation of lamellae and disruption of their organization appeared in cultures of $C$. fritschii grown at high light intensities. They also reported that algae grown in darkness were generally found in the endospore (coccoid) rather than the filamentous stage. This was in agreement with observation on darkgrown and light-grown Nostoc muscorum A (Lazaroff \& Vishniac, I96I).

The study reported here differs from previous work in that the ultrastructural phenomena can be examined in relationship to the developmental cycle of the organism, even though the morphological development is under the influence of light.

\section{METHODS}

The inocula for cultures were prepared in the following way: Nostoc muscorum A was grown in the light on Medium I (Lazaroff \& Vishniac, I96I) containing I \% sucrose. It was cultured in Erlenmeyer flasks on a shake machine and continuously illuminated with coolwhite fluorescent light at an intensity of $166 \mathrm{ft}$-candles. When the shake-grown material was two to three weeks old, it consisted mainly of actively motile hormogonia, which were suit- 
able for use as inocula. The alga was recovered aseptically by centrifugation at $438 \mathrm{~g}$. The supernatant was decanted, and the algal material, suspended in sterile Medium I, was blended for $\mathrm{I} O \mathrm{~s}$ in a Waring microblender. The blended preparation was washed three times by centrifugation, decantation, and resuspension in sterile Medium I. Drops of the resulting suspension were used to inoculate screw-cap tubes containing I I ml of Medium I with sucrose. The tubes were then placed in a light-tight box for dark incubation at $23{ }^{\circ} \mathrm{C}$.

Dark-grown cultures were incubated for 19 and 58 days, then harvested and fixed within an hour of their removal from darkness. These procedures were carried out while the organisms were shielded from the light with aluminium foil.

Additional cultures which had been dark-grown for 19 days were exposed to cool-white fluorescent illumination at an intensity of $100 \mathrm{ft}$-candles. Samples of these were fixed for electron microscopy after two, four and six days in the light. In addition, a culture grown for 2 I days on a shake machine illuminated with cool-white fluorescent light at an intensity of I $66 \mathrm{ft}$-candles, was harvested and fixed for electron microscopy.

The fixative was $5 \%$ unbuffered $\mathrm{KMnO}_{4}$ (Mollenhauer, 1959), at room temperature for $8 \mathrm{~min}$, followed by three to five rinses with water. The fixed material was embedded in $2 \%$ agar for ease of handling, cut into small blocks, and dehydrated through $25 \%, 50 \%, 75 \%$ and $95^{\circ}$, ethanol for 15 -min periods with two changes of solution. This was followed by $\mathrm{I} h$ in absolute ethanol and $\mathrm{I} h$ in propylene oxide. The resin used was Luft's Epon 8I 2 mixture (Luft, I96I) and contained six parts of mixture A to four parts of mixture B with $1.5 \%$ DMP 30 accelerator. The embedding was completed by transferring the material to fresh resin mixture in plastic capsules and allowing the blocks to harden for three days at increasing temperatures of $35^{\circ} \mathrm{C}$ to $60^{\circ} \mathrm{C}$. Sections were cut with glass knives on a Porter Blum microtome, then mounted on copper grids, and examined with a Philips Ioo electron microscope.

\section{RESULTS}

Before fixation of the algae, each developmental stage was observed with the light microscope (Fig. I). The material which had been incubated in darkness for 19 days was in the aseriate condition. The algae illuminated for two days after dark incubation could not be distinguished from the dark-grown material by light microscopy. After four days in the light, heterocystous filaments of large, round cells were visible within the packets. After six days in cool-white illumination, the filaments had elongated, and consisted of slender rectangular cells. These filaments had not broken at the heterocysts to form hormogonia. The algae grown in shake culture for $2 \mathrm{I}$ days at a light intensity of $\mathrm{I} 66 \mathrm{ft}$-candles consisted almost entirely of motile trichomes and detached heterocysts. The culture incubated in darkness for 58 days contained packets of aseriate cells and some short chains of round or ovoid cells with attached heterocysts.

Electron micrographs of thin sections of the material dark-grown for 19 days exemplified the ultrastructure of aseriate cells with compressed shapes embedded in a matrix of sheath material (Fig. 2). Heterocysts were observed both outside and within the packets (Fig. 2, 3). Those heterocysts outside the packets were believed to be the differentiated terminal cells of the parent trichomes; those within the packets were thought to be partially differentiated cells which might form the intercalary heterocysts of the filaments.

The ultrastructure of the aseriate cells was similar to that of vegetative cells of various blue-green algae (Pankratz \& Bowen, 1963; Wildon \& Mercer, 1963; Lang, I965; Peat \& Whitton, 1967). A layered wall and an undulating plasma membrane bounded each cell (Fig. 3). The granular inclusions were the cyanophycin granules, $\alpha$ granules, $\beta$ granules, 

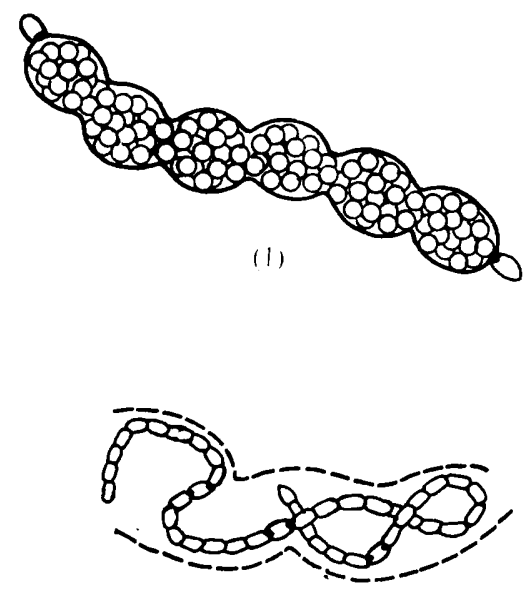

(3)

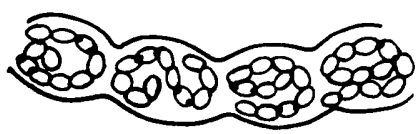

(2)

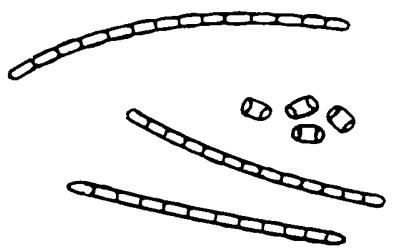

(4)

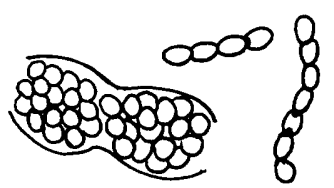

(5)

Fig. I. Diagram of changes in morphology of Nostoc muscorum a before fixation for electron microscopy. (1) Aseriate morphology exhibited by organisms maintained in darkness for 19 days, as well as those subsequently illuminated for two days. (2) Cellular arrangement within developing trichomes after four days of illumination. (3) Coiled filaments within degenerating sheath material after six days of illumination. (4) Motile hormogonia and detached heterocysts of shake-grown culture. (5) Aseriate packets and short trichomes of a dark-grown culture incubated for 58 days.

and polyhedral bodies. The cyanophycin and $\beta$ granules were generally seen in parietal positions while the polyhedral bodies were located in the central region of the cell. The $\alpha$ granules appeared to be concentrated around the cytoplasmic lamellae or thylakoids (Fig. 3).

The thylakoids of filamentous blue-green algae have generally been observed as parallel stacks or whorls of lamellae in the parietal region of the cell (Lang, 1968). In this study, the dark-grown cells contained a profuse array of lamellae frequently arranged in convoluted parallel pairs (Fig. 2). Such thylakoids were not confined to the peripheral region of the cell, but occupied all but a relatively small centroplasmic area.

Heterocysts found in aseriate material were recognizable because of their enlarged cell envelopes (Fig. 2, 3). Their cytoplasmic contents were similar to that of vegetative cells except that they appeared to lack cyanophycin granules and polyhedral bodies.

Although morphological changes could not be observed with the light microscope after the dark-grown material had been illuminated for two days, electron microscopy revealed features differing from those of the unilluminated packets. The cells appeared to be less compressed within the sheath material, and some were clearly in serial arrangement. The cytoplasmic matrix of this preparation seemed to be composed mainly of $\alpha$ granules (Fig. 4), but polyhedral bodies, cyanophycin granules, and $\beta$ granules were also observed as in the dark-grown algae. The lamellar system of the illuminated algae differed markedly from that 

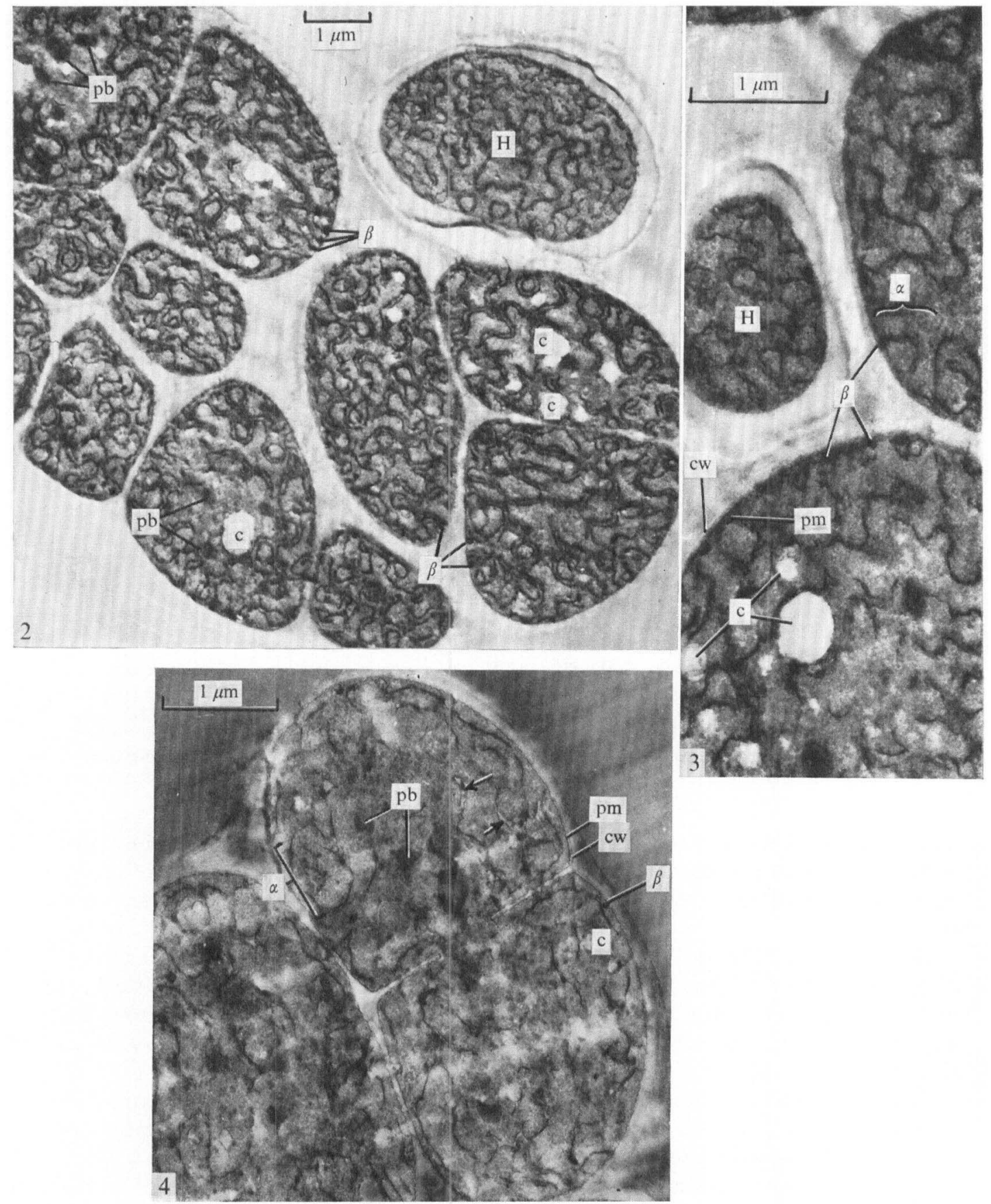

Fig. 2. Section through aseriate cells of Nostoc muscorum A dark-grown for I 9 days. Note compressed shapes of cells and the homogeneous arrangement of single and paired convoluted lamellae. Subcellular features include parietally located $\beta$ granules $(\beta)$ as well as the cyanophycin granules (c) and polyhedral bodies $(\mathrm{pb})$. A heterocyst $(\mathrm{H})$ appears outside the cell packet.

Fig. 3. Section of dark-grown aseriate cells with newly differentiated heterocyst $(H)$ within cell packet. Plasma membrane $(\mathrm{pm})$ and wall $(\mathrm{cw})$ bounded individual cells. Alpha particles $(\alpha)$ can be seen dispersed throughout the cytoplasm.

Fig. 4. Section of cells illuminated for two days after dark incubation. Note invagination of plasma membrane $(\mathrm{pm})$ and wall $(\mathrm{cw})$ in dividing cell. Granular inclusions present are cyanophycin granules (c), polyhedral bodies (pb), $\alpha$ granules $(\alpha)$, and $\beta$ granules $(\beta)$. Photosynthetic lamellae are sparser than those in unilluminated cells and exhibit disarray, fragmentation and vesiculation (arrows). 
of the dark-grown material. Thylakoids were less abundant, more fragmented, more vesiculated and were less frequently observed in convoluted patterns.

After four days in the light, the sections exhibited a filamentous algal arrangement. The lamellae of these cells were more abundant than those in the material illuminated for two days and tended to be restricted to the parietal region. Parallel pairs of thylakoids had again formed. Heterocysts observed at this stage contained a reticulate lamellar configuration (Fig. 5).

After six days of illumination the algal cells were more elongated (Fig. 6). Lamellae were seen in parallel parietal stacks of four and five thylakoids and in whorled configuration. Heterocysts at this stage retained a reticulate lamellar system (Fig. 6) and appeared to lack large granular inclusions.

The filaments of Nostoc muscorum A which developed from the aseriate stage were similar in ultrastructure to light-grown hormogonia which had been produced in shaken cultures. The cells of the hormogonia were longer and thinner than those of non-motile filaments which had developed after six days in the light (Fig. 7). Thylakoids of the hormogonia were found in the whorled arrangement rather than as the parietal stacks seen frequently in filaments developing after six days in the light. Detached heterocysts found in the preparation of hormogonia displayed a vacant cytoplasm and a disarrayed fragmented lamellar system similar to the structure described by Wildon \& Mercer (I963).

After 58 days in darkness, the cells were still present mainly within packets, and in most cases appeared similar to younger dark-grown aseriate cells. However, serial arrangement of cells could be observed in some formations. It appeared that this preparation had slowly begun to differentiate filaments in darkness. This was in agreement with the observations of Lazaroff (I966) and Robinson \& Miller (1970), who reported slow development of filaments in Nostoc muscorum A and $N$. commune during prolonged dark incubation. The progress toward filamentous morphology varied within this older dark-grown material as did lamellar configuration. Some cells contained curved parallel pairs of thylakoids similar to younger dark-grown cultures, while others contained curved single lamellae (Fig. 8), and still others exhibited parallel parietal layers of lamellae (Fig. 9). In some sections parietal lamellar rings comprised the entire lamellar complement. Generally, the aseriate cells exhibited the pattern of curved lamellae uniformly dispersed in the cytoplasm, while the more filamentous aggregates contained parallel parietal lamellae. During division, thylakoids were moved from their parietal position by the advancing septum, which pushed the lamellae before it and ultimately cut through them.

Heterocysts with thickened walls were seen more frequently in electron micrographs of the older dark-grown material (Fig. 8). Their lamellar configuration did not differ markedly from neighbouring vegetative cells, implying incomplete development to mature heterocysts.

\section{DISCUSSION}

Ultrastructural differences between I9-day-old dark-grown aseriate cells and light-grown cells of filaments and hormogonia of Nostoc muscorum a were manifested by variations of lamellar configuration. The thylakoids of the aseriate cells were found in curved pairs and were arranged almost homogeneously throughout the cytoplasm, allowing for only a small centroplasmic region devoid of lamellae. In contrast, the thylakoids of the seriate cells were generally concentrated in parietal regions of the cytoplasm, and stacks or whorls of three, four, and five parallel lamellae were frequently observed. The type of lamellar configuration exhibited by aseriate cells of $N$. muscorum A had previously been reported in dark-grown cells 

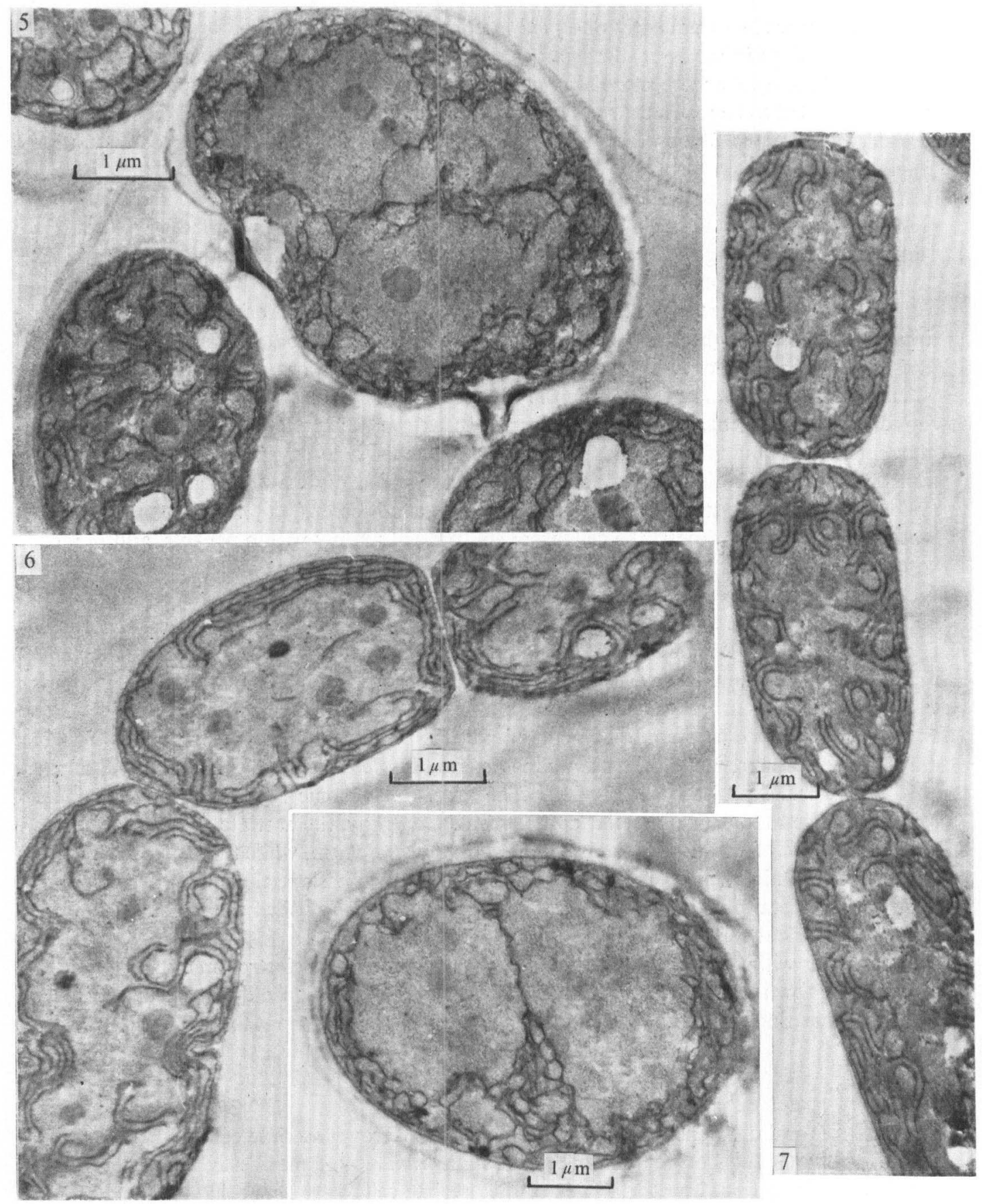

Fig. 5. Section through filamentous Nostoc muscorum A with an intercalary heterocyst. Material had been illuminated for four days after dark incubation. Heterocyst contains lamellae in reticulate arrangement.

Fig. 6. Longitudinal section through filament of Nostoc muscorum A after six days of illumination, showing parallel and whorled arrangement of parietally located lamellae.

Inset: Heterocyst after six days of illumination with reticulate lamellar configuration and lack of large granular inclusions.

Fig. 7. Longitudinal section through cells of light-grown hormogone of Nostoc muscorum A. Cells are more slender than those of light-induced filaments. Lamellar arrangement contains whorls rather than the parallel parietal stacks of light-induced filaments. 

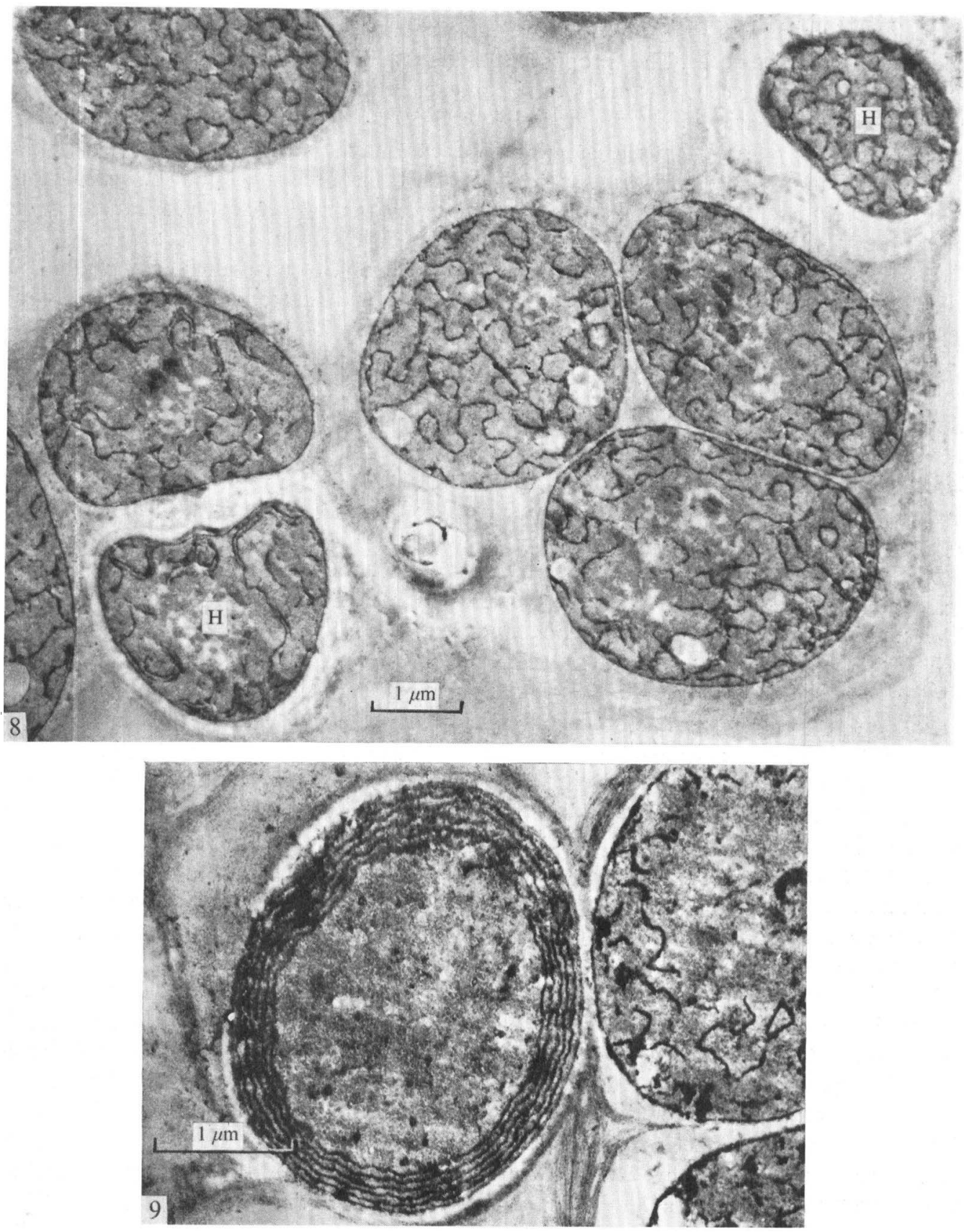

Fig. 8. Section through 58-day-old dark-grown aseriate cells displays the most frequently observed dark-grown cell morphology. The lamellae are single or paired and are uniformly dispersed throughout the cytoplasm. Two heterocysts $(\mathrm{H})$ within the packets display cytoplasmic features similar to those of vegetative cells.

Fig. 9. Section through cells of 58-day-old dark-grown Nostoc muscorum A. Total lamellar content of cell at left is arranged in parallel parietal rings. Inner lamellae are absent. 
of Chlorogloea fritschii by Peat \& Whitton (1967). It was suggested by those authors that the extension of thylakoids into the central region was a consequence of the increase in cell volume of the aseriate cells. They proposed that the requirement for biosynthetic substances throughout the larger cells was met by the homogeneous arrangement of thylakoids.

The striking similarity in ultrastructure between dark-grown cells of Nostoc muscorum A and Chlorogloea fritschii tends to support the idea that the pattern of cell and trichome development in these two organisms is homologous. It has been suggested (Lazaroff, I972) that the developmental pattern observed in $N$. muscorum a may have evolved from a similar but abbreviated life cycle exemplified by $C$. fritschii (Fay, Kumar \& Fogg, I964).

The appearance of the lamellae in cells illuminated for two days after dark incubation differed from lamellar arrangements of both aseriate and filamentous cells. It is as yet undetermined whether the fragmented, vesiculated thylakoids bear any intrinsic relationship to cell filament differentiation. The observation that dark-grown cells of the 58 -day-old culture, arranged in short chains, did not possess similar phases of lamellar alteration, precludes an unequivocal correlation of the lamellar changes with normal filament differentiation. The question remains whether exposure to light brought about the lamellar changes independently of the process of filament formation.

A similar phenomenon has been observed in cells of Chlorogloea fritschii (Peat \& Whitton, 1967; Findley et al. 1970).

The sparseness of lamellae in sections of material illuminated for two days may have resulted from the rapid production of cytoplasmic material which ensues after exposure to light and results in dilution of the lamellar content.

The 58-day-old dark-grown material exhibited a variety of lamellar patterns. Some were similar to the I9-day-old aseriate cells while others had configurations of closely appressed thylakoids which were parietally located and arranged in parallel stacks and rings. The parallel stacks closely resembled structures observed in old cultures of Symploca muscorum (Pankratz \& Bowen, 1963) which were interpreted as collapsed membranous regions devoid of $\alpha$ granules. However, with Nostoc muscorum A, these structures were most commonly observed in cells that had become arranged in short chains. It was thought that these parietal membranes had been newly synthesized at the plasma membrane, and were analogous to the parietal lamellae found in light-grown filaments and hormogonia. The absence of whorled arrangements may have been a consequence of the slow development of these filaments and/or the non-photosynthetic role of the lamellae in darkness.

The electron microscope revealed heterocysts within packets of the I9-day-old aseriate material which had not been distinguishable with the light microscope. These heterocysts were thought to be partially differentiated cells which might have become the fully differentiated intercalary heterocysts of the filaments. Perhaps the heterocysts in the aseriate material were analogous to the proheterocysts of Anabaena ambigua described by Bahal \& Talpasayi (1970) which were only recognizable when staining demonstrated their lack of polyphosphate granules. In that study, the partially differentiated cells had the capacity to revert to vegetative function and structure after the addition of fixed nitrogen.

This project was supported by Grant GM 10576, National Institute of General Medical Sciences, U.S. Public Health Service. 


\section{REFERENCES}

Allen, M. M. (1968). Photosynthetic membrane system in Anacystis nidulans. Journal of Bacteriology $\mathbf{9 6}$, $836-84 \mathrm{I}$.

Bahal, M. \& Talpasayi, E. R. S. (1970). Control of heterocyst development in Anabaena ambigua Rao. First International Symposium on the Taxonomy and Biology of Blue-green Algae. University of Madras, India.

FAY, P., Kumar, H. D. \& FogG, G. E. (1964). Cellular factors affecting nitrogen fixation in the blue-green alga Chlorogloea fritschii. Journal of General Microbiology 35, 35I-360.

Findley, D. L., WALNE, P. L. \& Holton, R. W. (I970). The effects of light intensity on the ultrastructure of Chlorogloea fritschii Mitra grown at high temperature. Journal of Phycology 6, $182-188$.

LANG, N. J. (1965). Electron microscopic study of heterocyst development in Anabaena azollae Strasburger. Journal of Phycology 1, 127-134.

Lang, N. J. (1968). The fine structure of blue-green algae. Annual Review of Microbiology 22, $15-46$.

LAzARoff, N. ( 1966). Photoinduction and photoreversal of the nostocacean developmental cycle. Journal of Phycology 2, 7-17.

Lazaroff, N. (1972). Photomorphogenesis and nostocacean development. In Biology of the Blue-green Algae. Edited by N. Carr and B. Whitton. Oxford: Blackwell Scientific Publications.

Lazaroff, N. \& Vishniac, W. (I96I). The effect of light on the developmental cycle of Nostoc muscorum, a filamentous blue-green alga. Journal of General Microbiology 25, 365-374.

LUFT, J. H. (1961). Improvements in epoxy resin embedding methods. Journal of Biophysical and Biochemical Cytology 9, 409-4I4.

Mollenhauer, H. H. (1959). Permanganate fixation of plant cells. Journal of Biophysical and Biochemical Cytology 6, $43 \mathrm{I}-436$.

Pankratz, H. S. \& Bowen, C. C. (1963). Cytology of blue-green algae. I. The cells of Symploca muscortum. American Journal of Botany 50, 387-399.

Peat, A. \& Whitton, B. A. (1967). Environmental effects on the structure of the blue-green alga, Chlorogloea fritschii. Archiv für Mikrobiologie 57, 155-1 80.

Robinson, B. L. \& Miller, J. H. (1970). Photomorphogenesis in the blue-green alga Nostoc commume 584 . Physiologia plantarum 23, 46I-472.

Wildon, D. C. \& Mercer, F. V. (I963). The ultrastructure of the heterocyst and akinete of the blue-green algae. Archiv fiir Mikrobiologie 47, 19-3I. 